Instituto Internacional de Investigación y Desarrollo Tecnológico Educativo INDTEC, C.A.

DOI: https://doi.org/10.29394/Scientific.issn.2542-2987.2019.4.14.5.101-119

OAI-PMH: http://www.indteca.com/ojs/index.php/Revista Scientific/oai

Artículo Original / Original Article

\title{
Psicología Organizacional como Componente Determinante de la Gerencia Interdisciplinaria
}

\author{
Autor: Erivan José Rondón Valero \\ Universidad de los Andes, ULA \\ erivan.rondon@gmail.com \\ Mérida, Venezuela \\ https://orcid.org/0000-0002-2170-001X
}

Resumen

Los seres humanos, como elemento social, son los responsables de las organizaciones de cualquier tipo a lo largo de la historia, son ellos los protagonistas de su propia evolución originada por sus intereses como necesidades de sobrevivencia. Por lo tanto, el papel protagónico de la Psicología Organizacional, es primordial, por ser la encargada de estudiar tanto el comportamiento individual como el grupal del SER. En ese sentido, se presenta como propósito general analizar la Psicología Organizacional como componente determinante de la gerencia interdisciplinaria del siglo XXI con la finalidad de acercarse a la comprensión del papel protagónico del hombre en su respectivo puesto de trabajo y su relación con sus compañeros laborales. En este sentido se considera el estudio desde el diseño de la investigación documental con enfoque cualitativo buscando, aproximarse al acercamiento de la compresión del principal autor de la película de la evolución del mundo holístico, complejo y globalizado. Por lo tanto, como principal reflexión investigativa se resalta la importancia de no seguir observando al trabajador como máquina, al contrario, es un ser de emociones: tristeza, alegría, las cuales afectan significativamente la productividad y competitividad de las organizaciones. La Psicología organizacional es responsable del logro de las metas organizacionales planificadas. humano.

Palabras clave: psicología; organización; comportamiento; ser

Cómo citar este artículo:

Rondón, E. (2019). Psicología Organizacional como Componente Determinante de la Gerencia Interdisciplinaria. Revista Scientific, 4(14), 101-119, e-ISSN: 2542-2987. Recuperado de: https://doi.org/10.29394/Scientific.issn.2542-2987.2019.4.14.5.101-119

Fecha de Recepción: 11-05-2019
Fecha de Aceptación:

09-08-2019
Fecha de Publicación:

05-11-2019 


\title{
Organizational Psychology as a Determinant Component of Interdisciplinary Management
}

\begin{abstract}
Human beings, as a social element, are responsible for organizations of any kind throughout history, they are the protagonists of their own evolution caused by their interests as survival needs. Therefore, the leading role of Organizational Psychology is essential, being the one in charge of studying both the individual and group behavior of the $\boldsymbol{B E}$. In that sense, it is presented as a general purpose to analyze Organizational Psychology as a determining component of the interdisciplinary management of the 21st century in order to approach the understanding of the leading role of man in his respective job and his relationship with his coworkers. In this sense, the study from the design of documentary research with a qualitative approach is considered, approaching the approach of the compression of the main author of the film of the evolution of the holistic, complex and globalized world. Therefore, the main research reflection highlights the importance of not continuing to observe the worker as a machine, on the contrary, it is a being of emotions: sadness, joy, which significantly affect the productivity and competitiveness of organizations. Organizational Psychology is responsible for achieving the planned organizational goals.
\end{abstract}

Keywords: psychology; organization; behaviour; human being.

How to cite this article:

Rondón, E. (2019). Organizational Psychology as a Determinant Component of Interdisciplinary Management. Revista Scientific, 4(14), 101-119, e-ISSN: 2542-2987. Recovered from: https://doi.org/10.29394/Scientific.issn.2542-2987.2019.4.14.5.101-119

Date Received:

11-05-2019
Date Acceptance:

09-08-2019
Date Publication:

05-11-2019 


\section{Introducción}

El ser humano (Hombre/Mujer), representa el principal activo productivo y competitivo de las organizaciones interdisciplinaria compleja del mundo actual, donde los gerentes modernos deben de brindarle las mejores condiciones laborales que permita lograr los más alto niveles de producción en la organización. Es importante destacar, que la Psicología Organizacional es la encargada de estudiar cuál es la mejor fórmula que permita aumentar el desempeño de los trabajadores, además de permitir la presencia del mejor clima organizacional.

Por lo tanto, es necesario que la gerencia de cualquier empresa bien sea del sector público o privado le otorgue el papel protagónico que representa esta rama de la psicología, la cual se especializa en el comportamiento del ser intrínsecamente en su puesto de trabajo, además en su relación con sus compañeros laborales.

Según Danvila y Sastre (2010), afirman que: "las emociones de los trabajadores pueden influir sobre el rendimiento de su trabajo" (pág. 120). Donde la Psicología Organizacional como factor determinante de las organizaciones complejas, debe de brindar las herramientas necesarias que permita controlar las conmociones de cada uno de los protagonistas en su jornada laboral. Glorifiquemos, que el Hombre es un ser de pasiones y no debe ser tratado como máquina acotando que el creador de todas esas nuevas tecnologías, equipos como maquinaria es el propio ser.

En este mismo orden de ideas, la dinámica global de la productividad y competitividad del mundo económico es marcado por ese fenómeno denominado "globalización" donde el mismo marca el compás de quienes permanecerá en el mundo globalizado y quienes no permanecerá, resaltando que su principal activo, sintiera que pasará a un segundo plano olvidando que sin ellos no existiría nada de lo que hoy podemos observar en este mundo dinámico como complejo. 
Se destaca la ineficiencia y las incapacidades para manejar los hechos surgidos de las estructuras y de las organizaciones sociales en la que los gerentes como principales representantes y líderes dentro de los procesos de cambio, podrán solucionar problemas sociales en función de satisfacer las necesidades de una sociedad, cubierta por la complejidad causada por el mismo, es por ello que se plantea: primeramente la implementación de un enfoque psicológico, al estudio de los problemas que existen en el mundo social y que a todos nos envuelve. En ese sentido, para Katz y Kahn (2008), quienes afirman que: "es un hecho elemental que las sociedades, debido a su naturaleza variada, representan agrupamientos organizados de personas cuyas actividades están institucionalmente encauzadas" (pág. 9); y en segundo lugar un enfoque desde la perspectiva de la gerencia interdisciplinaria para la formación y desarrollo gerencial promoviendo y fortaleciendo así el saber integral, a través de la interdisciplinariedad, la cual le permitan a los generes modernos un abordaje significativo y una amplia visión del todo, en función de orientar a la organización hacia horizontes de competitividad.

El propósito de la presente investigación es realizar una articulación critica de cómo la aplicación adecuada de la Psicología organizacional afecta significativamente en la productividad como la competitividad de las organizaciones complejas interdisciplinaria, por lo que metodológicamente se fundamenta en el enfoque cualitativo con base al diseño de investigación documental realizada por medio de diferentes investigadores que presenta reflexiones o resultados sobre el tema.

\section{El Problema}

Se ha observado en las organizaciones modernas del siglo XXI la necesidad de contestar a las exigencias competitivas del entorno, originado y marcada por el compás del fenómeno de la globalización que para bien o para mal a todos nos afecta. Dese este criterio de la investigación, se orienta a 
reflexionar ¿Cómo y Por qué? la psicología organizacional en el entorno complejo interdisciplinario actual, no se le otorga la credencial protagónica que la misma debería asumir, si por medio de ella se puede acercar a la compresión del comportamiento individual como organizacional del ser, además de la penuria en el privilegio del enfoque de trabajo en equipo, el énfasis al clima organizacional, en el manejo en lo que es la gerencia del cambio, el enfoque en la comunicación al desarrollo de la creatividad, la importancia del liderazgo, y en énfasis lo que es la motivación.

$\mathrm{Si}$ bien, un factor importante son las necesidades que un individuo necesita cubrir, no solo referida a las necesidades relacionadas a las básicas (alimentación, educación, recreación, habitacional); también aquellas como el respeto, el saludo, reconocimiento en el desempeño laboral del individuo, recibir afecto, y aspectos tanto emocionales como sentimentales que las personas necesitan satisfacer, que de alguna u otra manera pueden estar relacionadas con el comportamiento organizacional, que coadyuvan como herramienta a los logros de los objetivos de la organización.

No obstante, garantizar la mejor compresión del principal activo productivo y competitivo de las organizaciones complejas interdisciplinaria, garantizará la obtención de las metas planificadas, en el corto, mediano y largo plazo. En ese sentido, se sigue observando la carrera competitiva que existe, pero olvidando a su director de orquesta titular, quien a la final colocará el toque armónico para el mejor cierre de la interpretación de la obra central. El hombre es el creador de su propia complejidad a lo largo de su historia, quizás originada por su propia subjetividad e individualismo que por naturaleza tienen, recordando que la Psicología organizacional es el componente determinante de la gerencia interdisciplinaria.

\subsection{Propósito de la Investigación}

De acuerdo a la fundamentación del impacto que representa el talento 
humano como factor determinante de las organizaciones moderna del siglo XXI y con la intención de realizar aportes significativos al conocimiento, se presenta como propósito de la investigación analizar el papel protagónico de la psicología organizacional como componente determinante de la gerencia interdisciplinaria.

\section{Referentes Teóricos}

\subsection{Psicología Organizacional}

La Psicología Organizacional en basé a Rucci (2008), citado por Aamodt (2010): la define como la encargada de "aumentar la dignidad y el desempeño de los seres humanos, así como de las organizaciones para las que trabajan al avanzar en la ciencia y conocimiento del comportamiento humano" (pág. 24). De acuerdo con lo antes planteado por el autor, esta rama de la ciencia de la Psicología se especializa específicamente en el estudio del desempeño de los trabajadores dentro de la organización, además de cómo lograr la mayor integración con la organización para la que trabajan. Queda evidenciado que los seres humanos representan el protagonista de la novela actual de la competitividad como la productividad, donde la compresión adecuada en su comportamiento dentro de sus puestos trabajo es fundamental. Por lo tanto, la gerencia interdisciplinaria debe de incorporar dentro de sus estudios organizacionales, el papel protagónico que representa la Psicología Organizacional, la cual se encargará de mantener la mejor comunicación, motivación, liderazgo, que permitirá obtener los mejores resultados esperados en el corto, mediano y largo plazo; además de permitir la mejor integración entre los trabajadores y los directivos que ayudará en crear un clima organizacional apropiado.

Derivado de lo antes expuesto, la psicología organizacional es establecida con el propósito de estudiar el comportamiento del individuo y su relación con los compañeros de trabajo, buscando la mayor integración posible 
que permita obtener el máximo rendimiento productivo. En este contexto, para el psicólogo organizacional Furnham (2001), citado en Muñoz y Mesa (2017), habla de la Psicología Organizacional como:

El estudio de la forma en que las personas se reclutan, seleccionan y socializan en las organizaciones; de la manera en que son recompensadas y motivadas; de la forma en que las organizaciones están estructuradas formal e informalmente en grupos, secciones y equipos, y de cómo surgen y se comportan los líderes. También analizan la manera en la cual las organizaciones influyen en los pensamientos, sentimientos y comportamientos de todos los empleados a través del comportamiento real, imaginario o implícito de los demás en su organización (pág. 10).

Es menester determinar que la psicología organizacional, es una ciencia que se encarga de estudiar la conducta, el comportamiento y las experiencias del ser humano en un contexto laboral y organizacional, desde una visión tanto individual como colectiva, las cuales aparecen cotidianamente en el ámbito laboral; su propósito fundamental es mejorar sus niveles de desarrollo personal, productividad, eficiencia, desempeño, calidad, mejoramiento continuo, entre los trabajadores y sus gerentes, desde una perspectiva de estudiar la conducta del ser humano y sus experiencias en el contexto laboral y organizacional, pero al mismo tiempo resolver problemas que aparecen bajo este contexto.

Asimismo, destaca la importancia de diagnosticar las diferentes áreas y aspectos que comprende la psicología organizacional como: comunicación, clima, estructura y procesos, condiciones de trabajo, cultura, liderazgo, motivación, negociación, resistencia al cambio (gerencia del cambio), acoso laboral, salud y enfermedades organizacionales, afecciones emocionales, que unen lo social y lo organizacional dentro de su entorno, impactando el desempeño y la satisfacción laboral de los trabajadores en una organización, los cuales son los aspectos que se abordaran en la presente investigación. 
Por ello, en una organización todo cambio constituye una modificación en las relaciones laborales e interpersonales cotidianamente, así como los hábitos, actitudes y comportamientos de todas las personas que conforman la organización, este cambio involucra variaciones en el día a día del ambiente laboral, que generalmente pasa inadvertido e ignorados por los trabajadores, sin embargo, cuando se produce un cambio organizacional de gran magnitud, esto es, cuando se sobrepasa el límite de sensibilidad de las personas, atrae su atención y produce cambios a nivel afectivo, emocional y psicológico, causando preocupación, ansiedad, miedo, incertidumbre, aflicción, frente a una situación nueva y desconocida, entonces se origina cierta reacción y resistencia frente a esos cambios que ocurren o deben ocurrir en la organización, los cuales serán parte del estudió del departamento de Psicología Organizacional.

No realizarlo de la manera adecuada, representa sinónimo de fracaso y el no cumplimiento de los objetivos planificados, que en un momento determinado cualquier organización se planté. Por lo tanto, la Psicología Organizacional es la ciencia actual que estudia el SER desde lo interno, y como se desempeña en su puesto de trabajo, además de la relación con sus compañeros de trabajo.

En este sentido, se trata de un concepto holístico que recibe aportes de otras áreas de la psicología: psicología clínica, psicología educativa, psicología social, psicología del deporte y actividad física, psicología jurídica y criminológica, neuropsicología, psicología de la salud, entre otros, así como también el aporte de la gerencia interdisciplinaria que comprende la administración de la organización desde distintos ámbitos como la sociología, la ética, la economía.

Del mismo modo, a medida que el ser humano se ha civilizado a través de la historia ha sentido la necesidad de relacionarse con otros seres humanos y por consecuencia ha sentido la necesidad de comunicarse de una forma 
apropiada. Actualmente se puede observar como las diferentes organizaciones se comparan con el ser humano, esto es que, las organizaciones tienen una personalidad jurídica, la cual se adquiere debido a su cultura organizacional, sin embargo lo que se correspondería tomar en cuenta principalmente es la necesidad de tener una comunicación eficiente y eficaz para así lograr todos sus objetivos, actividades, tanto organizacionales como de todos aquellos que conforman su recurso más valiosos y fundamental que es el ser humano.

\subsection{Organizaciones Complejas}

Hay toda una temática a indicar que las organizaciones de cualquier tipo humanas o no son complejas por naturaleza, característica originada por su biósfera tanta externa como interna. Por lo tanto, la complejidad representa una característica fundamental presente a lo largo de la historia de la humanidad, su estudio de forma cuidadosa, permitirá mayor compresión del ser en su propio ámbito natural, recordando que, por medio de la teoría de las relaciones humanas, teoría de la complejidad y de sistemas abierto, se logrará mayor acercamiento a la compresión del ser como protagonista de su propia historia este sentido, Rodríguez (2016), expresa:

Mirar a las organizaciones con la visión de la complejidad permite comprender su dinámica y comportamiento. La organización es la emergencia producto de las interrelaciones y retroacciones de los elementos que la compone, y de éstos con el entorno cambiante y dinámico. La organización es caos y orden, es impredecibilidad y estabilidad (pág. 102).

Con respecto a lo antes mencionado, las estructuras de las organizaciones vistan desde la premisa de la complejidad son complejas por naturaleza, la cuál es originada por el propio ser, originado por el comportamiento de las personas y grupos que la conforman. Además, cada uno de ellos por naturaleza son complejos. Por lo tanto, cada organización es 
diferente, y puede adoptar la estructura organizacional que se adecue a sus prioridades, necesidades y requerimientos.

No obstante, es importante señalar que el Paradigma de la Complejidad ha sido desarrollado por Edgard Morin entre los años 1976 y 1988, donde el autor desarrolla un estudio titulado Introducción al Pensamiento Complejo. Para Morin (1998): "la complejidad no es una receta para conocer lo inesperado. Pero nos vuelve prudentes, atentos, no nos deja dormirnos en la mecánica aparente y la trivialidad aparente de los determinismos" (pág. 75). Se evidencia, que la complejidad constituye un medio para abordar la creación del conocimiento como la comprensión de los fenómenos de la humanidad, la vida, la existencia, el desarrollo humano, la sustentabilidad del ser humano y por ende del desarrollo organizacional, aunado a esto dentro del pensamiento complejo se rompe con la concepción de un método rígido y racional, establecido en estándares que limitan al ser humano.

Por lo tanto, la presencia de las organizaciones complejas es un hecho real, donde la Gerencia Interdisciplinaria debe tomarla como otro elemento integrador de la compresión del crecimiento y desarrollo de las organizaciones modernas del siglo XXI. No tomarlo en cuenta, seria sinónimo de fracaso o simplemente se estaría estacionando en la simplificación de la realidad; recordando que ese mismo ambiente organizacional no es estático, al contrario, es dinámico, cambiante, integrador, holístico y complejo.

La visión de organizaciones complejas es causada por el propio ser, el cuál es subjetivo como individualista. En tal sentido, desde los fundamentos teóricos que permitirá la mayor integración holística, es necesario el estudio y compresión de la teoría del Caos, teoría de las relaciones humanas, teoría de sistemas abierto las cuáles permitirá la integración prospectiva de las organizaciones complejas con enfoque en la gerencia interdisciplinaria en la modernidad del siglo XXI. 


\subsection{Gerencia Interdisciplinaria}

El abordaje teórico, se fundamenta según Rodríguez (2015), en que:

Lo relevante de este planteamiento de la Gerencia está en la posibilidad de que pueda ser abordada desde una perspectiva interdisciplinaria, en la cual el dominio de estudio va más allá del terreno disciplinar hasta ahora reservado exclusivamente a la administración. En tal sentido, de lo que se trata es de organizar una epistemología particular con los conocimientos aportados en este caso por distintas disciplinas y ciencias como la sociología, la psicología, la economía, la política y la administración por su puesto, contemplando en este recorrido como hilo conductor las reflexiones del fundamento ético y moral (pág. 95).

En este contexto, los paradigmas gerenciales se han desarrollado en consonancia con las exigencias y transformaciones del entorno empresarial globalizado, para garantizar la autorregulación de las organizaciones, así como el alcance de una mayor eficiencia de sus objetivos organizacionales estratégicos. Estos cambios y transformaciones del siglo XXI, se caracterizan por el aumento de la competencia, el avance tecnológico, la alta rotación de productos, la evolución constante de las necesidades de consumo, la innovación, las telecomunicaciones, la digitalización, la cibernética, como otros elementos han llevado a las organizaciones a replantear sus objetivos y metas.

No obstante, se ha ignorado un área de gran importancia como lo es la conducta del ser humano en las organizaciones, en base a lo planteado en la teoría de las relaciones humanas. En consecuencia, se ha cambiado supuestos en el medio empresarial, a fin de señalar las distintas perspectivas que pueden adoptar las organizaciones ante procesos de cambio y transformación.

En tal sentido, surge una nueva concepción de la gerencia orientado hacia la formación del gerente, destacando que se debe considerar su visión y prospectiva en el cambiante y competitivo mundo de la gerencia, que es cada 
vez más exigente y competitiva; en este enfoque se presenta la perspectiva integradora de una serie de variables y fenómenos sociales, que permiten el estudio de todos los factores que participan en el proceso de formación y desarrollo de gerentes ante los nuevos retos, destacando la importancia de la epistemología en el mencionado proceso, en procuras de lograr una visión global y ampliada, en conjunto con otras ciencias, que proporcionen al gerente de hoy, herramientas que le permitan responder a las exigencias continuas de la organización, por lo tanto, el conocimiento requerido se aprecia de una manera extensa, debido a la transformación de la realidad organizacional, acotando que cada organización responde a un contexto específico.

Consecuentemente, los procesos de cambios, son una respuesta que ofrecen las organizaciones, de forma espontánea a las incertidumbres o transformaciones del entono, pues debe entenderse que el carácter sistémico de las mismas condicionan también para su supervivencia; deben generar interacciones con el micro y el macro ambiente, obteniendo los recursos idóneos que requieren para el desarrollo de sus productos o servicios los cuales respondan a las expectativas de clientes, consumidores y por ende al de la sociedad.

La gerencia de hoy, implica disponer de las capacidades teórico prácticas con las cuales disponen, para enfrentar los cambios tecnológicos, enfocando todos los esfuerzos en el logro de la integración de los recursos humanos, materiales, tecnológicos y financieros, que permitan la producción de bienes o servicios de calidad, apuntalada primordialmente en herramientas tecnológicas con visión de integración, así como también la capacitación constante del personal encargado de motorizar las áreas que integran la organización.

En este contexto, las organizaciones son integración de los líderes o gerentes con todos los integrantes que conforman la estructura, con la meta de dar cumplimiento a los objetivos planificados de forma conjunta. Es por ello 
por lo que, la Psicología Organizacional como elemento determinante en la complejidad de la gerencia interdisciplinaria, los líderes dentro de su distribución deben estar alineados para la toma de decisiones de forma vinculada con toda la planta laboral, con el objetivo de lograr un ambiente armónico.

En este contexto, la gerencia de Cambio, hacia la Perspectiva de la Gerencia Interdisciplinaria del Siglo XXI, representan que las organizaciones cada día apuntan hacia nuevos enfoques en su dirección, planificación y estructuración, debido al fenómeno de la globalización existente, así como al ambiente competitivo en el que se desenvuelven. No obstante, los gerentes toman en consideración un proceso de cambio, de pasar de un estado organizacional a otro, sin embargo, el mismo puede ser positivo o negativo, el cual debe orientar en que se realice un cambio positivo en beneficio de los trabajadores y por ende a la organización. En tal sentido, Chiavenato (2002a), define: "cambio significa pasar de un estado a otro. Es la transición de una situación a otra. El cambio implica transformación, perturbación, interrupción y ruptura, que dependen de su intensidad" (pág. 150).

De acuerdo a lo planteado por el autor, esto nos conlleva a la siguiente interrogante ¿cómo debe ser el cambio gerencial? Debe ser flexible y tranquilo, se considera un cambio en lo externo, como lo es las diferentes políticas, practicas, o estructuras que el líder o gerente este tratando de implementar no autoritario ni acelerado; y un cambio a lo interno, refiriéndose a la reorientación psicológica a la cual se someten los trabajadores antes que el cambio se lleve a cabo. En base a Ivancevich, Konopaske y Matteson (2012a), afirman que: "el cambio es una condición saturante, persistente y permanente de todas las organizaciones" (pág. 579).

En este mismo orden de ideas, los gerentes pueden iniciar el cambio organizacional en diversas formas, como lo expresan Ivancevich, Konopaske y Matteson (2012b), al decir que la: 
Administración del cambio por medio del poder: la aplicación del poder para producir el cambio implica coacción. El administrador tiene acceso al poder y con él coacciona para dirigir el cambio como él desea (...) en consecuencia, por su acceso a este poder, los administradores pueden ejercer considerable influencia en una organización. El ejercicio del poder con frecuencia se manifiesta en un liderazgo autocrático, y las organizaciones contemporáneas en general no alientan a los administradores a tener tal comportamiento (...) (pág. 582).

En base a lo anteriormente presentado esto es, la administración de cambio de la gerencia por medio de la reeducación generalmente comprende el desarrollo organizacional, el cual sin duda afirma que es la estrategia normativa, que produce cambio en el proceso cognitivo del individuo, siendo su fin último el agente del cambio, asimismo Ivancevich, Konopaske y Matteson (2012c), expresan que:

El desarrollo organizacional es un proceso que genera el cambio en un sistema social, lo cual plantea la cuestión del agente de cambio (el individuo o grupo que se convierte en el catalizador del cambio (...) se reconoce que el cambio organizacional entraña cambios sustanciales en los pensamientos, creencias y acciones de los individuos (pág. 582).

También para Chiavenato (2002b): "agente de cambio es la persona (de adentro o fuera de la organización) que conduce o guía el proceso de cambio en una situación organizacional. Puede ser un miembro de la organización o un consultor externo" (...) (pág. 151).

En este contexto, es importante, que se produzca el cambio organizacional, debido que se requiere necesariamente involucrar a todas las personas que se debe hacer en equipo tanto las gerencias como todos los trabajadores, para que funcionen como agentes activos y no pasivos del cambio que se pretende impulsar. En opinión de Chiavenato (2002c), afirma: "el cambio organizacional debe ser planeado, organizado, dirigido y controlado 
con mucha dirección. En otros términos, debe ser administrado con una visión estratégica que privilegie la totalidad de la organización y el futuro de la empresa y del negocio" (pág. 161).

En relación con los cambios organizacionales, existen cuatro (4) clases, Chiavenato (2002d), establece: 1). Cambios en la estructura organizacional; 2). Cambios en la tecnología; 3). Cambios en los productos o servicios: afectan los resultados o salidas de la organización; y 4). Cambios en las personas 0 en la cultura de la organización (pág. 159).

Por ello, en una organización todo cambio constituye una modificación en las relaciones laborales e interpersonales cotidianamente, así como los hábitos, actitudes y comportamientos de todas las personas que conforman la organización, este cambio involucra variaciones en el día a día del ambiente laboral, que generalmente pasa inadvertido e ignorados por los trabajadores, sin embargo, cuando se produce un cambio organizacional de gran magnitud, esto es, cuando se sobrepasa el límite de sensibilidad de las personas, atrae su atención y produce cambios a nivel afectivo, emocional y psicológico, causando preocupación, ansiedad, miedo, incertidumbre, aflicción, frente a una situación nueva y desconocida, entonces se origina cierta reacción y resistencia frente a esos cambios que ocurren o deben ocurrir en la organización.

Con respeto a lo antes planteado, el papel protagónico de la Psicología organizacional es fundamental, es ella la encargada de dirigir todos los cambios que son necesarios en las organizaciones, originado por las exigencias cambiantes y permanente que las organizaciones complejas envueltas por el fenómeno de la globalización deben necesariamente realizar para poder permanecer en el campo competitivo y productivo de la gerencia interdisciplinaria moderna del siglo XXI. 


\section{Referente Filosófico y Epistemológico}

La teoría de las relaciones humanas representa el fundamento epistemológico de la Psicología Organizacional, la cual se originó por medio de los estudios desarrollados por Elton Mayo (1880-1949), la cual tiene su plena aplicabilidad y desarrollo en las organizaciones modernas, complejas e interdisciplinarias que conforma la gerencia de hoy. Sus aportes científicos en el factor humano dentro de las organizaciones son fundamental y por medio de ella, se puede obtener una mejor aproximación a la compresión del ser.

\section{Marco Metodológico}

El enfoque de la presente investigación está fundamentado en el cualitativo. Para Martínez (2006), afirma: "trata de identificar, básicamente, la naturaleza profunda de las realidades, su estructura dinámica, aquella que da razón plena de su comportamiento y manifestaciones" (pág. 66). De acuerdo a lo planteado por el autor, la investigación cualitativa está basada en el hecho de que cada ser humano es diferente desde cualquier ámbito en el que se desempeñe llámese social, cultural e ideológico, por tal razón el paradigma cualitativo abarca el estudio de cada ser humano de acuerdo al contexto donde se desenvuelve, tomando en cuenta sus conductas, limitaciones, opiniones, emociones, actitudes e interpretaciones de la realidad y del mundo que los rodea, los cuales determinan su conducta ante la sociedad. En relación a lo antes planteado, el diseño de la investigación corresponde al tipo documental.

\section{Reflexiones del Investigador}

La Psicología Organizacional es el componente determinante de la competitividad y productividad de las organizaciones interdisciplinarias modernas del siglo XXI, es necesario que los gerentes de hoy le otorguen el papel protagónico que está ciencia desempeña en su principal activo como lo es el SER (hombre/mujer). Recordemos que el creador de todos los grandes 
inventos y adelanto que ocurren y han ocurrido en la humanidad es él, quien siente la necesidad como la creatividad de darle respuesta a todas las necesidades que en su evolución experimenta permanentemente. No debemos olvidar que las organizaciones al final de todo están conformado por seres humanos quienes no son máquinas humanas, al contrario, persistentemente experimenta emociones como tristeza, alegría, preocupaciones, temor, duda las cuales afecta directamente en la obtención y el logro que las organizaciones en un tiempo determinado se plantean como meta. El conocimiento es infinito, y solo el protagonista de su propia novela tendrá la respuesta en cada uno de los próximos capítulos que se espera desarrollar en la infinita historia cambiante y prospectiva de la humanidad. La Psicología organizacional es la herramienta principal de la Gerencia moderna, compleja e Interdisciplinaria del mundo globalizado actual, dejarla a un lado es sin duda algún sinónimo de fracaso organizacional.

\section{Referencias}

Aamodt, M. (2010). Psicología Industrial/Organizacional: Un Enfoque Aplicado. 6ta. Edición, ISBN 10: 607-481-346-9; ISBN 13: 978-607481-346-3. México, D.F.: Cengage Learning Editores, S.A. de C.V. Recuperado de:

https://cucjonline.com/biblioteca/files/original/a1bdc9466890694ff0a11 e937b269278.pdf

Chiavenato, I. (2002a,b,c,d). Administración en los nuevos tiempos. ISBN 10: 9584103016; ISBN 13: 9789584103017. Bogotá, Colombia: McGraw-Hill.

Danvila, I., \& Sastre, M. (2010). Inteligencia Emocional: Una revisión del concepto y líneas de investigación. Cuadernos de estudios empresariales, (20), 107-126, e-ISSN: 1131-6985. Recuperado de: https://dialnet.unirioja.es/servlet/articulo?codigo=3934705 
Furnham, A. (2001). Psicología Organizacional. El comportamiento del individuo en las organizaciones. México: Editorial Oxford.

Ivancevich, J., Konopaske, R., \& Matteson, M. (2012a,b,c). Comportamiento Organizacional. México: McGraw-Hill.

Katz, D., \& Kahn, R. (2008). Psicología social de las organizaciones. México, D.F.: Editorial Trillas.

Martínez, M. (2006). Ciencia y arte en la metodología cualitativa. México: Editorial Trillas.

Morin, E. (1998). Introducción al pensamiento complejo. México, D.F.: Multiversidad Mundo Real Edgar Morin. Recuperado de:

http://cursoenlineasincostoedgarmorin.org/images/descargables/Morin Introduccion al pensamiento complejo.pdf

Muñoz, J., \& Mesa, I. (2017). Diagnóstico de clima organizacional en una planta de derivados cárnicos. Trabajo de grado. Medellín, Colombia: Universidad de Antioquia. Recuperado de:

http://200.24.17.74:8080/jspui/handle/fcsh/840

Rodríguez, J. (2016). Las Organizaciones Complejas. Ingeniería e Investigación, (50), 90-103, e-ISSN: 2248-8723; ISSN: 0120-5609 Recuperado de:

https://revistas.unal.edu.co/index.php/ingeinv/article/view/21428

Rodríguez, M. (2015). La gerencia interdisciplinaria. Lo natural y lo humano. 1ra. Edición, ISBN: 978-980-7453-15-8. Valencia, Venezuela: Instituto de Previsión Social del Personal Docente y de Investigación IPAPEDI.

Rucci, A. (2008). I-O psychology's "core purpose": Where science and practice meet. The Industrial-Organizational Psychologist, 46(1), 1734. 


\section{Erivan José Rondón Valero}

e-mail: erivan.rondon@gmail.com

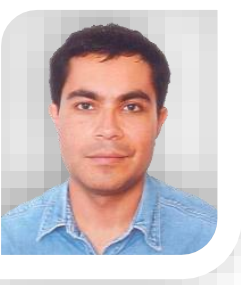

Nacido en Venezuela, el 23 de noviembre del año 1983.

Economista de Universidad de Los Andes (ULA); Licenciado en Educación Mención Básica Integral de la Universidad Católica Cecilio Acosta (UNICA); Postgrado en Tributos, Mención Rentas Internas de la Universidad de Los Andes (ULA); Componente Docente Básico, en Educación Superior de la Universidad de Los Andes (ULA); Doctor en Gerencia Avanzada de la Universidad Fermín Toro (UFT); Investigador Activo en la Universidad de Los Andes (ULA); Planificador de la Dirección General de Planificación y Desarrollo en la Universidad de Los Andes (ULA); Profesor Invitado a nivel de pregrado, postgrado y Doctorado de la Universidad Fermín Toro (UFT); Universidad Pedagógica Experimental Libertador (UPEL); Universidad Nacional Experimental de Los Llanos Occidentales Ezequiel Zamora (UNELLEZ); y la Universidad Nacional Experimental de la Seguridad (UNES); Asesor y jurado de trabajos de investigación de las universidades ULA; UFT; y UPEL.

El contenido de este manuscrito se difunde bajo una Licencia de Creative Commons ReconocimientoNoComercial-Compartirlgual 4.0 Internacional 